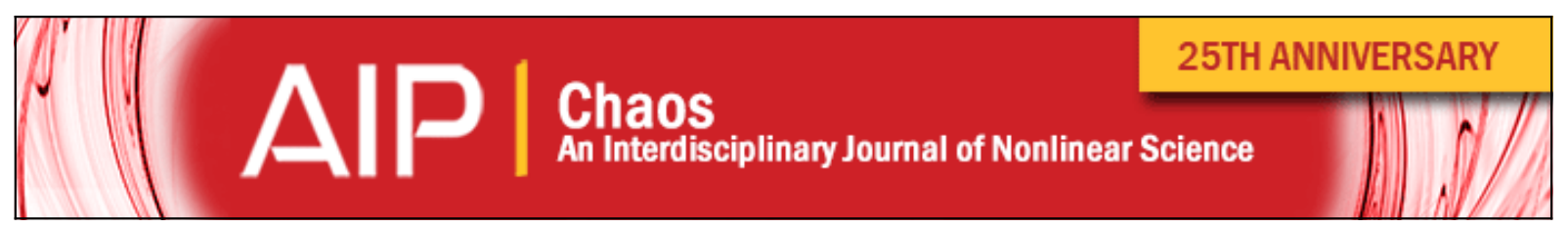

\title{
Controlled generation of switching dynamics among metastable states in pulse- coupled oscillator networks
}

Hai-Lin Zou, Yuichi Katori, Zi-Chen Deng, Kazuyuki Aihara, and Ying-Cheng Lai

Citation: Chaos 25, 103109 (2015); doi: 10.1063/1.4930840

View online: http://dx.doi.org/10.1063/1.4930840

View Table of Contents: http://scitation.aip.org/content/aip/journal/chaos/25/10?ver=pdfcov

Published by the AIP Publishing

\section{Articles you may be interested in}

Metastability and chimera states in modular delay and pulse-coupled oscillator networks

Chaos 22, 043131 (2012); 10.1063/1.4766592

Reconstructing phase dynamics of oscillator networks

Chaos 21, 025104 (2011); 10.1063/1.3597647

Breakdown of order preservation in symmetric oscillator networks with pulse-coupling

Chaos 21, 025113 (2011); 10.1063/1.3589960

Oscillations in Coupled Systems: Response to a Noise-Contaminated Signal

AIP Conf. Proc. 922, 473 (2007); 10.1063/1.2759723

Experimental investigation of high-quality synchronization of coupled oscillators

Chaos 10, 738 (2000); 10.1063/1.1286996

\section{Cross-pollinate.}




\title{
Controlled generation of switching dynamics among metastable states in pulse-coupled oscillator networks
}

\author{
Hai-Lin Zou, ${ }^{1}$ Yuichi Katori, ${ }^{2,3}$ Zi-Chen Deng, ${ }^{1,4}$ Kazuyuki Aihara, ${ }^{2}$ and Ying-Cheng Lai ${ }^{5}$ \\ ${ }^{1}$ School of Mechanics, Civil Engineering and Architecture, Northwestern Polytechnical University, \\ Xi' an 710072, China \\ ${ }^{2}$ Institute of Industrial Science, University of Tokyo, 4-6-1 Komaba, Meguro-ku, Tokyo 153-8505, Japan \\ ${ }^{3}$ The School of Systems Information Science, Future University Hakodate, 116-2 Kamadanakano-cho, \\ Hakodate, Hokkaido 041-8655, Japan \\ ${ }^{4}$ School of Science, Northwestern Polytechnical University, Xi' an 710072, China \\ ${ }^{5}$ School of Electrical, Computer and Energy Engineering, Arizona State University, Tempe, Arizona 85287, USA
}

(Received 8 April 2015; accepted 31 August 2015; published online 16 September 2015)

\begin{abstract}
Switching dynamics among saddles in a network of nonlinear oscillators can be exploited for information encoding and processing (hence computing), but stable attractors in the system can terminate the switching behavior. An effective control strategy is presented to sustain switching dynamics in networks of pulse-coupled oscillators. The support for the switching behavior is a set of saddles, or unstable invariant sets in the phase space. We thus identify saddles with a common property, localize the system in the vicinity of them, and then guide the system from one metastable state to another to generate desired switching dynamics. We demonstrate that the control method successfully generates persistent switching trajectories and prevents the system from entering stable attractors. In addition, there exists correspondence between the network structure and the switching dynamics, providing fundamental insights on the development of a computing paradigm based on the switching dynamics. (C) 2015 AIP Publishing LLC.

[http://dx.doi.org/10.1063/1.4930840]
\end{abstract}

Exploiting nonlinear dynamical systems for applications in information science and technology has been a fascinating area of research. The paradigm of associative memory pioneered by Hopfield is one example. Another example is chaos-based computing that makes use of a large number of different dynamical "states" inherent to the system for encoding and processing information. Recently, heteroclinic switching dynamics in networks of nonlinear oscillators have been proposed as a potential class of devices for information encoding, with significant implications to biological systems. In particular, a large number of saddles (or unstable attractors) can emerge, which constitute the backbone for dynamical switching. Information encoding can be achieved by making the system visit the neighborhoods of these saddles and switch among them from time to time. Many combinations of saddles offer a rich variety of possible switching patterns. However, the appearance of stable attractors can terminate heteroclinic switching dynamics. Without control, there is a high probability that the system, starting from a random initial condition, will approach a stable attractor. Utilizing networks of pulse-coupled oscillators as prototypes of a class of systems, we develop an effective localization control strategy to govern the system from one saddle to another without ever falling into the basin of any stable attractor. Our key idea lies in making use of a class of saddles with common local properties, which provide a platform to execute the localization control to direct the network dynamics to switch from one saddle to another. As a result, the natural trajectories that link these saddles are not needed to be discovered as the localization control substitutes them. We uncover correspondence between the network structure (e.g., various loop structures) and switching dynamics, allowing the global switching dynamics to be harnessed through certain collective behaviors emerging among subsets (or groups) of oscillators.

\section{INTRODUCTION}

Exploiting nonlinear dynamical behaviors for information processing and computing has a long history (see, for example, Refs. 1-12). The celebrated Hopfield associative memory devices, a class of artificial neural networks, make use of nonlinear response functions to store and retrieve information. ${ }^{1-3}$ The fundamental tasks involved in computing such as information storage, communication, and processing can all be accomplished by exploiting the flexibility of nonlinear dynamical systems - the paradigm of chaos computing. ${ }^{4-7}$ Especially, chaotic systems have extremely complex dynamical behaviors, but it is exactly this complexity that makes available a large number of different dynamical "states" that can be used for encoding information. In addition to human designed or engineered systems, nonlinearity has also been exploited in natural systems for information processing. For example, in systems biology, ${ }^{13}$ the Hills function, a nonlinear response function, is generic and fundamental to various gene regulatory networks.

A nonlinear system can exhibit a rich variety of dynamical invariant sets ${ }^{14}$ such as attractors and non-attracting chaotic sets responsible for transient chaos. ${ }^{15,16}$ A common type of simple invariant sets is saddles (more generally unstable 
periodic orbits). In fact, a chaotic invariant set has an infinite number of embedded unstable periodic orbits. ${ }^{14} \mathrm{~A}$ trajectory starting from a random initial condition visits the neighborhoods of these orbits from time to time- a kind of switching behavior that is the base for practical applications such as chaos control. ${ }^{17-19}$ In the past few years, there has been a great deal of effort in investigating switching dynamics among saddles in nonlinear networks from the perspective of information encoding. ${ }^{20-28}$ For switching dynamics among saddles, the system typically spends long time in the vicinity of a saddle, while the switching from one saddle to another occurs relatively fast. Due to the large number of combinations of saddles available, this approach has been deemed to be potentially useful for computation.

A dynamical approach to realizing switching is through heteroclinic connections, each joining together two different saddles, which corresponds to the unstable manifold of the starting saddle and the stable manifold of the terminal saddle. The resulting dynamics is effectively a type of heteroclinic switching dynamics. To realize heteroclinic switching, it is necessary to manipulate the saddles and their unstable manifolds. One approach to generating the heteroclinic switching dynamics is based on the principle of winnerless competition, where many metastable states such as saddles and trajectories connecting them are used to model sequential switching dynamics observed in some neural systems. ${ }^{20}$ In this regard, the saddles with one-dimensional unstable manifolds have been used to generate switching dynamics. ${ }^{29,30}$ When the unstable manifolds are high-dimensional, a trajectory starting from one saddle can approach multiple saddles, making it difficult to generate the desired switching dynamics, i.e., any single switching sequence of saddles where switching between two successive saddles is explicitly determined.

Besides the approach of winnerless competition, systems with symmetries have also been exploited for realizing heteroclinic switching dynamics. In such a system, multiple saddles could be connected cyclically, leading to stable heteroclinic cycles. ${ }^{31}$ In a globally pulse-coupled oscillator network with delayed excitatory coupling, heteroclinic switchings can occur, where the saddles are actually unstable attractors. ${ }^{32-34}$ These saddles form a complicated network of states in the phase space, and symmetry-breaking inhomogeneities can effectively induce heteroclinic switchings. ${ }^{24,25}$ When the networks are not globally connected, stable attractors can become dominant, which prevent the natural occurrence of heteroclinic switching dynamics.

Previous methods focused on generating switching dynamics through direct establishment of heteroclinic connections that are intrinsically present. In order to generate many switching patterns, a large number of saddles are required, which usually occur in large networks. However, as the network size increases, the task becomes extremely difficult because the number of conditions associated with the heteroclinic connections, as well as the number of distinct network structures and the dimensionality of the unstable manifolds of the saddle, increases rapidly. In addition, to realize switching dynamics, it is desirable that the system dynamics be simple, e.g., exhibiting saddles only, but chaotic dynamics can often emerge in large networks. ${ }^{35}$ For these reasons, previous effort was mainly devoted to switching dynamics in globally coupled networks or small networks. In addition, the correlation between network structure (or topology) and heteroclinic switching dynamics has not been well understood.

In this paper, we focus on the local dynamics of saddles and develop a control framework to generate desired switching dynamics to guide the system from one saddle to another. In this way, the difficult problem of establishing heteroclinic connections among saddles is avoided. We address this issue using networks of pulse-coupled oscillators, a paradigm for diverse natural systems such as neural networks, cardiac pacemaker cells, and flashing fireflies. ${ }^{36-45}$ Despite the simplicity of the individual oscillator dynamics, such a network (even of large size) exhibits a large number of interconnected saddles, or a "network" of saddles, and simultaneously a large number of stable attractors. To achieve efficient switching dynamics, the system's approaching any stable attractor should be avoided. We develop an effective approach to manipulating switching dynamics, taking advantage of the properties of the saddles and their local dynamical behaviors. Specifically, we find a type of saddles with prescribed properties that are directly related to the network structure. That is, by selecting the appropriate network structure, the system can potentially exhibit the desired switching dynamics. We articulate an effective localization strategy to restrict the state of the system to the neighborhoods of the saddles only so as to avoid being attracted toward any of the stable attractors. Our results yield a better understanding of the correspondence between network structure and switching dynamics, and it can be useful for the design of information processing and computing devices based on harnessing unstable saddle dynamics in coupled oscillator networks.

In Sec. II, we describe a type of saddles with common properties, and then design network structure accordingly. In Sec. III, we present our localization control method to generate desired saddle switching dynamics, bypassing the stable attractors. In Sec. IV, we demonstrate the effectiveness of our method using numerical examples. In Sec. V, we present conclusions.

\section{SADDLE PROPERTIES AND NETWORK STRUCTURE}

\section{A. Network of excitatory pulse-coupled oscillators}

A widely used model for a network of pulse-coupled oscillators was introduced by Mirollo and Strogat ${ }^{37}$ to understand the synchronous flashing behaviors of fireflies. This is essentially a model of integrate-and-fire oscillators in computational neuroscience. ${ }^{33}$ In a network of pulse-coupled oscillators, the state of the $i$ th oscillator is described by a phase-like variable $\phi_{i}(t) \in(-\infty, 1]$. The free evolution, i.e., the dynamics without interactions, is given by

$$
d \phi_{i} / d t=I_{i},
$$

where $I_{i}$ is phase velocity of oscillator $i$, chosen to be unity $\left(I_{i}=1\right)$. When $\phi_{i}(t)$ reaches the threshold 1 , oscillator $i$ fires and its phase $\phi_{i}(t)$ is reset to zero. This process is called 
firing or spiking, during which a pulse is generated. After a delayed time $\tau$, oscillators with incoming links from oscillator $i$ will receive the pulse. For example, when oscillator $j$ receives a pulse from oscillator $i$ at time $t$, a phase jump in oscillator $j$ is induced as follows:

$$
\phi_{j}^{+}(t)=\min \left\{U^{-1}\left[U\left(\phi_{j}(t)\right)+\varepsilon_{j, i}\right], 1\right\},
$$

where $\phi_{j}^{+}(t)$ is the phase of oscillator $j$ just after the pulse and $\varepsilon_{j, i}$ denotes the coupling strength from oscillator $i$ to $j$. The coupling strength is normalized as $\varepsilon_{j, i}=\varepsilon / k_{j}$, and $k_{j}$ is the in-degree of $j$, i.e., the number of incoming links. In other words, the total strength of the incoming links for an oscillator $j$ is $\sum_{i} \varepsilon_{j, i}=\varepsilon$. Generally, the directed connections cannot have the same strength, because the in-degree determines the individual connection strengths. Throughout this paper, we consider excitatory coupling with $\varepsilon_{j, i}>0$. A possible choice for $U(\phi)$ is ${ }^{37}$

$$
U(\phi)=b^{-1} \ln \left[1+\left(e^{b}-1\right) \phi\right],
$$

with a positive parameter $b$.

\section{B. Properties of saddles}

The two events associated with pulses, i.e., generation (denoted by $S$ ) and receipt of pulses (denoted by $R$ ), can be used to describe the properties of saddles. Pulses are indexed based on their origin. For example, $S(i)$ indicates when a pulse is generated by oscillator $i$, and $R(i)$ denotes the receipt of a pulse from oscillator $i$. When multiple oscillators have the same event, the notations are similar. For example, $S(i, j)$ indicates that oscillators $i$ and $j$ generate pulses at the same time, and $R(i, j)$ denotes the receipt of pulses from these two oscillators. Time durations in which no event occurs are denoted by the minus sign "-.".

In terms of the event sequences, we find a large number of saddles sharing the following common dynamical properties. Several oscillators reach the threshold simultaneously during the free evolution, i.e., simultaneously active firing, while other oscillators fire due to the arrival of supra-threshold pulses, the so-called passive firing. ${ }^{46}$ For the latter case, an oscillator with phase $\phi$ fires immediately when receiving a supra-threshold pulse with strength $\varepsilon^{\prime}$, i.e., $U(\phi)+\varepsilon^{\prime} \geq 1$. These saddles are in fact unstable attractors, which are locally unstable but with a positive measure of the basins of attraction $^{32-34,47,48}$ and hence are a type of Milnor attractors. ${ }^{49}$

A typical event structure of a saddle (an unstable attractor) is exemplified as

$$
R(1,2) S(3,4,6)-R(3,4,6) S(5)-R(5)-S(1,2),
$$

which specifies a sequence of events during one period for a saddle in a globally connected network of 6 oscillators. The events and the detailed evolution of the system are listed in Table I. In this example, the first three events are arrivals of pulses started by notation $R$. The last event is that oscillators 1 and 2 become simultaneously active firing $S(1,2)$. These two oscillators receive sub-threshold pulses and reach the threshold during free evolution. The saddles of structure (4) have the
TABLE I. For a system of six globally coupled oscillators, typical events for a saddle with the simultaneously active firing $S(1,2)$. The phases are recorded just after the occurrence of an event. Immediately after the first event, oscillators 3, 4, and 6 fire passively, and their phases are reset to zero: $R(1,2) S(3,4,6)$. After the delayed time $\tau=0.1$, the pulses from these three oscillators are received, causing oscillator 5 to fire passively: $R(3,4,6) S(5)$. The parameters are $\tau=0.1, \varepsilon=0.1$, and $b=1.5$.

\begin{tabular}{ccccccccc}
\hline \hline & & \multicolumn{7}{c}{ Phases of oscillators } \\
\cline { 5 - 9 }$k$ & Event & Time $t$ & 1 & 2 & 3 & 4 & 5 & 6 \\
\hline 1 & $R(1,2) S(3,4,6)$ & 20.06 & 0.11 & 0.11 & 0.0 & 0.0 & 0.89 & 0.0 \\
2 & $R(3,4,6) S(5)$ & 20.16 & 0.25 & 0.25 & 0.12 & 0.12 & 0.0 & 0.12 \\
3 & $R(5)$ & 20.26 & 0.37 & 0.37 & 0.23 & 0.23 & 0.1 & 0.23 \\
4 & $S(1,2)$ & 20.88 & 0.0 & 0.0 & 0.86 & 0.86 & 0.72 & 0.86 \\
5 & $R(1,2) S(3,4,6)$ & 20.98 & 0.11 & 0.11 & 0.0 & 0.0 & 0.89 & 0.0 \\
6 & $R(3,4,6) S(5)$ & 21.08 & 0.25 & 0.25 & 0.12 & 0.12 & 0.0 & 0.12 \\
7 & $R(5)$ & 21.18 & 0.37 & 0.37 & 0.23 & 0.23 & 0.1 & 0.23 \\
8 & $S(1,2)$ & 21.80 & 0.0 & 0.0 & 0.86 & 0.86 & 0.72 & 0.86 \\
\hline \hline
\end{tabular}

additional property, that is, the time difference between two successive arrivals of pulses, such as the second and the third events in Table I, is $\tau=0.1$. This property arises ${ }^{50}$ because of the absence of passive firings for the last arrival of the pulses (denoted as $R(5)$ in this example) before the simultaneously active firing $S(1,2)$. There also exist saddles where some passive firings are caused by $R(5)$. In such cases, the time difference between two successive arrivals of pulses is smaller than $\tau$, depending on the phases of the oscillators. This induces great difficulty to manipulate or control the corresponding saddles. We thus focus only on the cases where there is no passive firing induced by the last event of the arrival of pulses before simultaneously active firing occurs.

\section{Rare saddle connections in sparse networks}

The saddles of similar structure (4) form the backbone of the switching dynamics. Since the saddles are unstable attractors, it is possible to directly investigate the connections among them. Numerical verification of the nature of the unstable attractor can be found in Refs. 33 and 46. Two unstable attractors can form a heteroclinic connection in the sense that nearby points for one unstable attractor can lead to another unstable attractor. ${ }^{47,51}$ To this end, we measure the fraction $f_{h c}$ of initial conditions leading to unstable attractors forming heteroclinic connections. Such an initial condition first leads to an unstable attractor, but points in its neighborhood can lead to the other unstable attractors. We also measure the fraction $f_{s t}$ of initial conditions that lead to stable attractors. For a network of size $N$ with $m$ directed links, the density $\rho$ is defined as $\rho=m /[N(N-1)]$, where each directed link is placed between two oscillators randomly selected from the network. A globally coupled network without self-links has the maximum number of links $N(N-1)$.

Figure 1 shows $f_{h c}$ and $f_{s t}$ versus the density $\rho$. We see that $f_{h c}$ approaches zero quickly when $\rho$ is decreased, and the basins of the stable attractors dominate the phase space. This means that the connections among saddles become rare, due to the emergence of the stable attractors with increased sparsity. 


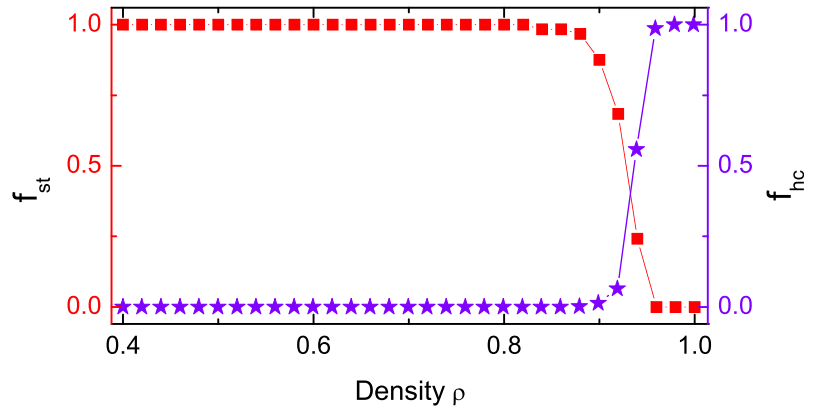

FIG. 1. The effect of density of links $\rho$ on the connections among saddles. Here, $f_{h c}$ is the fraction of initial conditions leading to unstable attractors forming heteroclinic connections, and $f_{s t}$ is the fraction of initial conditions leading to stable attractors. When density $\rho$ decreases, the saddles connections become rare with $f_{h c}$ approaching zero, due to the dominance of basins of the stable attractors with $f_{s t}$ close to unity. The data points are obtained by averaging over 100 random initial conditions. Other parameters are $N=30, b=1.5, \varepsilon=0.2$, and $\tau=0.03$.

\section{Networks with group structures}

Except the difficulty from rare saddle connections in generating switching dynamics, one needs to identify the type of networks that can generate saddles with simultaneously active firing. The topological criterion that the firing sequences require can be met by classification of oscillators into groups, where each group of oscillators receive the same set of incoming links, with no excitatory couplings within the group so as to exclude self-links. The network structure is schematically shown in Fig. 2(a). In this type of networks, a group of oscillators can sustain simultaneous firings through the receipts of either no pulse or pulses of the same strength, once they have been reset to the same phase.

We then consider the event properties of saddles in networks with groups. Generally, one group of oscillators become simultaneously active firing, while other groups fire passively. It is thus useful to regard each group of oscillators as one unit. Suppose there are $N_{g}$ number of groups, where the $i$ th group is represented by $G_{i}$. A general event structure for the unstable attractors in terms of groups is given by

$$
R\left(G_{1}\right) S\left(G_{i}\right)-R\left(G_{i}\right) S\left(G_{m}\right)-\cdots-R\left(G_{k}\right)-S\left(G_{1}\right),
$$

where oscillators in the group $G_{1}$ fire simultaneously and actively, i.e., $S\left(G_{1}\right)$. The time difference between two successive arrivals of pulses is $\tau$. Such event property in terms of groups facilitates analysis of the system's approaching a certain saddle through the establishment of the corresponding event structure.

The unstable nature of the saddle with simultaneously active firing in networks with groups can be reasoned, as follows. For a saddle, we first add small instantaneous perturbations on the phase variables just after the resetting of the reference oscillator, which introduces an initial phase difference for the originally simultaneously active firing oscillators. Here, the magnitude of the perturbations should be small enough, as the goal here is to explore the local dynamic behavior of the saddle. We show in the following that the phase difference grows as time goes by. Consider two active firing oscillators, $i$ and $j$, which receive pulses of the same strength, as they are in the same group. Suppose

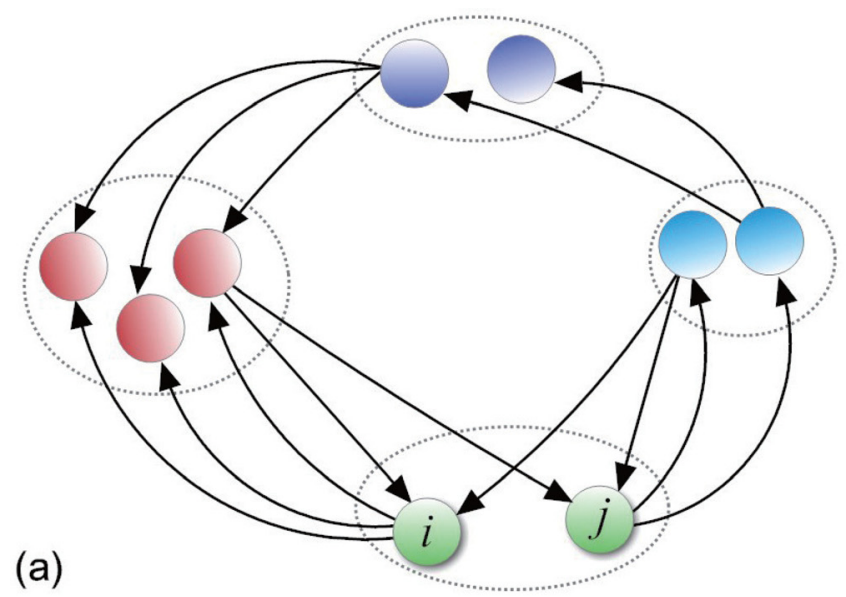

(b)

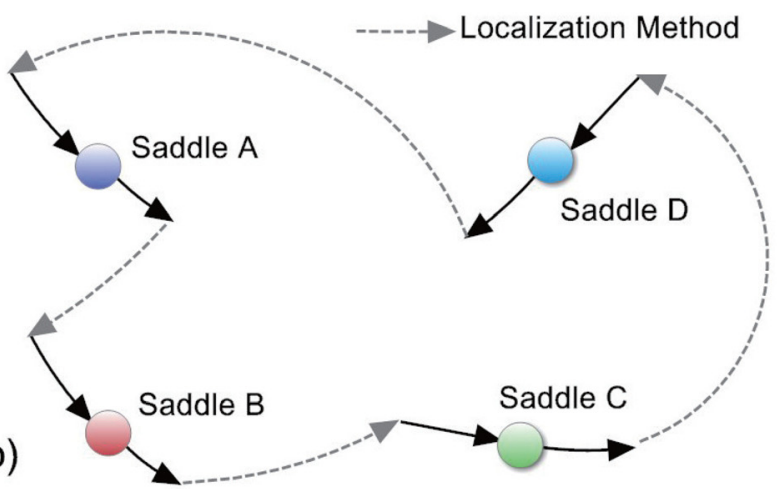

FIG. 2. Schematic illustration of network structure and switching dynamics. (a) Representation of a network structure, in which oscillators are divided into groups. Oscillators in the same group receive the same incoming excitatory coupling denoted by the directed links. (b) Generation of switching dynamics using localization control method that drives the state of the system to local regions of the saddles.

that they receive a pulse of strength $\varepsilon^{\prime}$ at time $t$. Infinitesimally prior to this event, the phase difference is $\Delta \phi_{i, j}(t)=\phi_{i}(t)-\phi_{j}(t)$. Immediately after the arrival of the pulse, the phase difference becomes

$$
\Delta \phi_{i, j}^{+}(t)=U^{-1}\left[U\left(\phi_{i}(t)\right)+\varepsilon^{\prime}\right]-U^{-1}\left(U\left(\phi_{j}(t)\right)+\varepsilon^{\prime}\right) .
$$

Substituting the expressions of $U$ and $U^{-1}$ into Eq. (6), we obtain

$$
\Delta \phi_{i, j}^{+}(t)=\exp \left(\varepsilon^{\prime} b\right)\left(\phi_{i}(t)-\phi_{j}(t)\right)
$$

Here, the saddles are of period one, i.e., each oscillator fires once and its pulse is received during one period, giving rise to the event structure (5). Thus, each oscillator receives all the pulses generated by its incoming neighboring oscillators during one period, with the total strength $\varepsilon$. The arrival of each pulse causes the phase difference to increase according to Eq. (7). We thus have

$$
\Delta \phi_{i, j}^{+}\left(t_{n+1}\right)=\exp (\varepsilon b) \Delta \phi_{i, j}^{+}\left(t_{n}\right),
$$

where $\Delta \phi_{i, j}^{+}\left(t_{n}\right)$ is the phase difference between oscillators $i$ and $j$ immediately after the $n$th resetting of the reference oscillator 1 at time $t_{n}$. We see that the phase difference increases, giving rise to the unstable dynamics within the active firing group. 


\section{E. Dynamics in networks with symmetrical group structures}

There are two types of symmetries at the group level. First, oscillators within the same group receive the same set of excitatory incoming links. Second, all oscillators are identical with the same phase velocities $I_{i}=1$. The existence of symmetry, together with the delayed excitatory connections, can induce various interesting dynamic behaviors, among which the emergence of unstable attractors is intriguing because such an "attractor" is in fact a saddle but with a remote basin of attraction. Stable attractors with surrounding basins of attraction can also arise.

The phase space can be divided into two parts, i.e., the basins of stable attractors and unstable attractors, where the density of links plays an important role in determining the whole basin size of each type of attractors. The basins of the unstable attractors can dominate the phase space for globally connected networks. ${ }^{32}$ In other cases, the basins of the stable attractors can be dominant, as shown in Fig. 1, rendering necessary application of control to generate switching dynamics. The appearance of stable attractors can terminate the switching dynamics. In Section III, we articulate our control strategy to prevent termination of switching dynamics.

\section{CONTROLLED GENERATION OF SWITCHING DYNAMICS}

For saddles of structures (5), oscillators in each group are identical and exhibit the same phase. In order to generate switching dynamics, it is necessary to break such symmetry by slightly modifying the phase velocities $I_{i}$ for $i=1,2, \ldots, N$. This can be done, for any oscillator $i$, by changing its parameter as $I_{i}=1+\delta_{i}$. Hence, we term $\delta_{i}$ as symmetry-breaking parameter, which is given by

$$
\delta_{i}=D \cdot A_{i} .
$$

Here, $A_{i}$ is randomly and uniformly chosen from the interval $[-0.5,0.5]$, and $D$ is the magnitude of the symmetrybreaking parameters. In order to split the simultaneously active firing of a group, we require that at least two oscillators in that group have different phase velocities. Otherwise, the system will stay on an unstable attractor indefinitely.

For small symmetry-breaking parameters, the original saddles of structure (5) become metastable states. It is insightful to analyze the effect of symmetry-breaking parameters on the event structure. In absence of symmetry breaking, a group with two oscillators, e.g., $i$ and $j$, becomes simultaneously active firing $-S(i, j)$, and oscillators in other groups fire passively. After applying the symmetry breaking parameters, the event $S(i, j)$ will be split into multiple active firings at slightly different times, such as $S(i)-S(j)$ for $\delta_{i}>\delta_{j}$. The dynamics of the passively firing groups are unaffected by the slight variation in phase velocities. The event property of the metastable state is then composed of one active firing group and other passive firing groups.

An obstacle to achieve switching dynamics is that stable attractors can become dominant in networks that are not globally connected. It is extremely difficult to directly establish the heteroclinic connections among the saddles, particularly for large networks with various structures. In this regard, a previous method of realization of heteroclinic switchings in globally connected network ${ }^{24}$ cannot be applied here, as it relies on the dominance of the unstable attractors.

\section{A. Localization control method}

We present a localization control method to overcome the above difficulties by harnessing the dynamics within the local regions of the metastable states. When localization control is applied, the system approaches a predefined metastable state spontaneously; then it spends some time in the metastable state, and before it leaves the metastable state, another localization control is applied, making the system approach another metastable state. Figure 2 (b) provides a schematic illustration of this process.

Our localization control method aims to rearrange the phases to force the system to achieve a particular metastable state of structure (5). The basic idea is to establish the specific events, where one group fires actively and others fire passively. For example, the arrival of pulses from some oscillators can directly induce passive firings of some other oscillators. This implies that there exist directed links from former oscillators to later oscillators, and former oscillators fire earlier than later oscillators. We thus place one group before another group if there are directed links from the former to the latter. To initiate localization control, we select one group as the group at the first level, which is also used to be the active firing group for the metastable state. The groups with incoming links from the first group are regarded as the groups at the second level. This process can be repeated to generate ordered levels of groups, until all groups have been selected.

The organization of groups into different levels is determined by the first level group and the connections among the groups. The metastable states of structure (5) imply that the passive firing groups are reachable from the active firing group through the directed links. In order to ensure that any group can serve as an active firing group for metastable states, we focus on the networks that are strongly connected in terms of groups; i.e., each group is reachable from every other group through the directed links. The resulting group levels depend on the choice of the first level group. For example, the groups in globally coupled networks can be organized into two levels: the first level group and all other groups in the second level.

According to the level structures of groups, the initial phase of an oscillator $i$ is written as

$$
\phi_{i}=1-\left(L e_{i}-1\right) c,
$$

where $L e_{i}$ denotes the oscillator $i$ 's level number and all oscillators in the same group have the same level number. The level number is $n$ for oscillators in groups at the $n$th level. The parameter $c$ describes the phase difference between the oscillators from the groups at two successive levels. 
How to choose the parameter $c$ ? The goal is to set up the event structure (5). After the firing of the groups at one level, the pulses will be received after delayed time $\tau$. In order to induce passive firing for the oscillators in the groups at the next higher level, the these oscillators should not fire before the arrival of pulses from the current level group. To keep such order, it is required $c>\tau$. We then consider the effect of $c$ on oscillators in the first level group, which are used to set up the events of active firings. For larger value of $c$, their phases are larger at the arrival of pulses coming from their neighboring oscillators, which is not good as the phases should be sufficiently small to avoid passive firings. Thus, the value of $c$ should be as small as possible. If $c$ is not small, the initial transients are going to be longer and even they may render the system in a different state than intended to be. Throughout this paper, $c=1.001 \tau$ is used.

We then consider the evolution of the system with the initial phase chosen according to Eq. (10). The oscillators in the first level group have phase $=1$, which fire actively immediately. The phase of an oscillator $i$ in the second level groups is $1-c$, due to the fact that the phase difference for the two successive groups is $c$. After a free evolution with time duration $\tau$, the pulses with strength $\varepsilon^{\prime}$ from the first level group are received. The phase of oscillator $i$ will gain the amount $\tau: \phi=1-c+\tau=1-0.001 \tau$. The passive firing of the oscillator $i$ requires $U(\phi)+\varepsilon^{\prime} \geq 1$. Under the condition $U(\phi)+\varepsilon^{\prime}>\phi+\varepsilon^{\prime}>1$, the oscillator $i$ becomes passive firing, which requires $\varepsilon^{\prime}>0.001 \tau$. This condition can readily be met when $\tau$ is small. The system will then gradually settle into the corresponding passive firing pattern enforced by the group order and form the event structure that one group fires actively while others groups fire passively. If this event structure can sustain for the current parameter setting, the chosen initial condition will lead to the metastable states with the first level group as the active firing group. An important issue is then in what parameter region do metastable states of structure (5) exist. This issue will be addressed in Sec. IV.

To generate switching between metastable states, it is necessary to apply localization control again to ensure that the system, after leaving the neighborhood of a metastable state, will not approach any stable attractor. The issue of timing, i.e., when to apply the control, is important. It is infeasible to address this issue for each individual metastable state. An alternative approach is to examine the leaving process for a number of metastable states, and to identify common dynamic behavior.

We find that the active firing group and the passive firing groups show different dynamic behavior when system leaves any metastable state. This property can be used to introduce the localization control. Specifically, we define the maximum phase difference for group $g$ as

$$
\Delta \phi_{g}=\max _{i, j \in g}\left[\min \left(\left|\phi_{i}-\phi_{j}\right|, 1-\left|\phi_{i}-\phi_{j}\right|\right)\right] .
$$

The maximum phase difference for the active firing group grows as $\exp (\varepsilon b)$ [see Eq. (7)]. Then, we consider the evolution of the maximum phase differences for passive firing groups under the small symmetry-breaking parameters.
When a group of oscillators begin to fire passively, the maximum phase difference for this group is zero. Symmetry breaking on $I_{i}$ can then introduce non-zero maximum phase difference for these passive firing groups, which can increase according to Eq. (6) temporarily at the arrivals of pulses, but becomes zero again due to the passive firing when the phases of all corresponding oscillators are simultaneously reset to zero. Thus, the maximum phase differences of passive firing groups are small, typically on the order of the magnitude of the symmetry-breaking parameters. As a result, the maximum phase difference for the active firing group reaches a relatively large value first, leading to a simple criterion to apply localization control

$$
\Delta \phi_{g_{a}}>d_{c}>\Delta \phi_{g_{o}}
$$

where $g_{a}$ denotes the group whose maximum phase difference becomes larger than $d_{c}$ first, and $g_{o}$ denotes all other groups. To be concrete, we fix $d_{c}=0.001$.

Once condition (12) is met, we apply the localization control, which is equivalent to selecting a group as the first level group with respect to the current metastable state. Various rules can be used to select the first level group. An intuitive rule is based on selecting the "winner," where an oscillator (or a group) with the largest phase velocity (or the largest average phase velocity) is chosen. For the current metastable state, we first select an oscillator with the largest phase velocity in the active firing group, i.e., the group with the maximum phase difference larger than $d_{c}$. The chosen oscillator is effectively the leading oscillator, as this oscillator will fire before other oscillators in the same group after application of localization control. Next, we choose one group with the largest average phase velocity from the groups with incoming links from the leading oscillator and designate this group as the first level group. Finally, we apply control to rearrange the phase of the system according to Eq. (10) by using the chosen first level group. The process is called localization control with winner-based rule.

\section{B. Simulation of system with localization control}

One advantage of pulse-coupled oscillators is that they can be simulated accurately and efficiently using the event driven approach, ${ }^{33}$ where the arrival of the pulse and oscillator's approaching its threshold are determined. To incorporate localization control into the simulation process, we monitor the maximum phase difference for each group right after every event (e.g., arrival of pulse or firing). We apply the control as soon as the system meets the condition (12). Let $\phi_{i}(t)$ represent the phase of oscillator $i$ at time $t$. Control can be computationally implemented, in the following steps:

(i) Locate the next firing time for the system due to the free evolution. The evolution of the phase for oscillator $i$ is $\phi_{i}\left(t^{\prime}\right)=\phi_{i}(t)+\left(t^{\prime}-t\right) I_{i}$, with time $t^{\prime}>t$. The firing time for oscillator $i$ is $t_{i}^{f}=\left[1-\phi_{i}(t)\right] / I_{i}+t$. The next firing time for the system is given by $t^{f}=\min _{i}\left(t_{i}^{f}\right)$.

(ii) Compare $t_{f}$ with the next arrival time of pulses $t_{a}$. If $t_{f}$ $<t_{a}$, proceed to step (iii); otherwise go to step (iv). 
(iii) The next event is reaching of the threshold for some oscillators. Update the phase as $\phi_{i}=\phi_{i}(t)+I_{i}\left(t_{f}-t\right)$ and update the time to $t=t_{f}$. Record the pulse when any oscillator reaches the threshold, reset its phase to zero, and go to step (v).

(iv) The next event is the arrival of pulses at time $t_{a}$. Update the phase due to the free evolution: $\phi_{i}=\phi_{i}(t)+I_{i}\left(t_{a}-t\right)$, and set the time to be $t=t_{a}$. Calculate the total strength of the received pulses for each oscillator $i$ and denote it as $\varepsilon_{i}$. If $U\left(\phi_{i}\right)+\varepsilon_{i}<1$, the phase of oscillator $i$ is $\phi_{i}=U^{-1}\left[U\left(\phi_{i}\right)+\varepsilon_{i}\right]$. Otherwise, reset the phase of oscillator $i$ to zero, so that a pulse is generated at time $t_{a}$, and then proceed to step (v).

(v) Incorporation of the localization control. (a) Measure the maximum phase difference of each group according to Eq. (12). (b) If only one group has its maximum phase difference larger than $d_{c}$, the system meets the condition (12). We then select a group as the first level group by using the winner-based rule, and prepare the initial condition according to Eq. (10). (c) Go to step (i).

\section{CONTROL PERFORMANCE}

The detailed construction of networks with groups is given in the Appendix. Throughout this paper, we consider two types of networks. For the first type, each oscillator points to only one group. In this case, the choice of the first level group is solely determined by the choice of the leading oscillator. For the second type, each oscillator $i$ has the same in-degree $k_{i}$, and each oscillator can point to multiple groups. We avoid the case that multiple oscillators in a group point to the same group, as the choice of different leading oscillators may affect the same group in this case. Thus, we have $k_{i} \leq\left(N_{g}-1\right)$ where $N_{g}$ is the number of groups. One particular example is the globally connected networks in terms of groups with $k_{i}=N_{g}-1$. In such case, each group is connected to all other groups.

\section{A. Generating switching dynamics through localization control: A detailed example}

The network has $N=30$ oscillators, organized into 11 groups, where each oscillator in any group point to only one group randomly selected from other groups. We set the magnitude of symmetry-breaking parameters $D=10^{-7}$.

We start simulating the system by randomly designating a group as the first level group to initiate localization control. Figure 3(a) shows a trajectory with switching dynamics among three metastable states, with active firing group 5, 3, and 6 , respectively. The evolution of the maximum phase differences for the three groups recorded just after the occurrence of every event is shown in Fig. 3(b), where we can see that localization control is triggered when the maximum phase difference for a group reaches the critical value $d_{c}=0.001$. Table II shows the detailed events and phases of oscillators from two groups 5 and 3 near the moment when the localization control is applied, inducing the first switching as shown in Fig. 3(a).

The groups selected by the leading oscillators form substructures for the switching patterns. For the three groups $(5,3$, and 6$)$, the leading oscillators and the directed connections among them are shown in Fig. 3(f). The three groups actually form a loop, where the leading oscillator in a former group points to the oscillators in a latter group. Accordingly, the system generates a stationary switching pattern among the three metastable states. Here, the loop structures at the group level directly determine the stationary switching patterns. In some cases, one may start with a group and visit a chain of groups following the directed links. As each oscillator has outgoing links, one finally settles into a loop. Accordingly, we observe a transient switching behavior among some metastable states determined by the chain, and finally reach the stationary switching pattern determined by the loop. In this sense, the loop structures are more important as they determine the stationary switching behaviors.

As the phases of the passive firing oscillators change slowly near a metastable state, only the phases of the active firing oscillators are relevant, which are in a lowdimensional subspace with the dimension being the number of the active firing oscillators. Then, the system sequentially visits some low-dimensional subspaces and jumps among these subspaces due to the localization control, as shown in Figs. 3(c)-3(e). The maximum phase difference of a group is $\phi_{i}-\phi_{j}$, where oscillator $i(j)$ has the largest (smallest) phase velocity $I_{i}\left(I_{j}\right)$. Then, the critical condition for (12) is $\phi_{i}-\phi_{j}=d_{c}$. For example, active firing group 5 has two oscillators 13 and 14, with $I_{13}>I_{14}$. The critical condition for (12) becomes $\phi_{13}-\phi_{14}=d_{c}$, which is shown as the green line in Fig. 3(c). Here, a part of the trajectory (1 to 53) is plotted where each point represents the phases of oscillators 13 and 14 at the resetting of the reference oscillator 1 . Figures 3(d) and 3(e) show the low-dimensional subspace dynamics for the second metastable state with active firing group 3 and the third metastable state with active firing group 6, respectively. Here, both of two groups 3 and 6 have three oscillators. A different oscillator can become the leading oscillator under a different symmetry-breaking parameters. Then, the system will leave the metastable state along a different direction but will still be in the same subspace. We thus plot the dynamics of metastable states in three dimensional subspaces, although the critical condition depends on the phases of two oscillators.

\section{B. Residence time and detection of metastable states}

The system can stay near a metastable state for relatively long time until the condition (12) is met. Here, we analyze the residence time $T_{s}$ of a metastable state, defined as the number of resettings of the reference oscillator between the start of localization control and the time when condition (12) is met. We choose two oscillators $i$ and $j$ in the active firing group with largest and smallest phase velocities, respectively. Then, the maximum phase difference for this group is just the phase difference of oscillators $i$ and $j$. The growth of the phase difference is due to two factors: local unstable dynamics and 

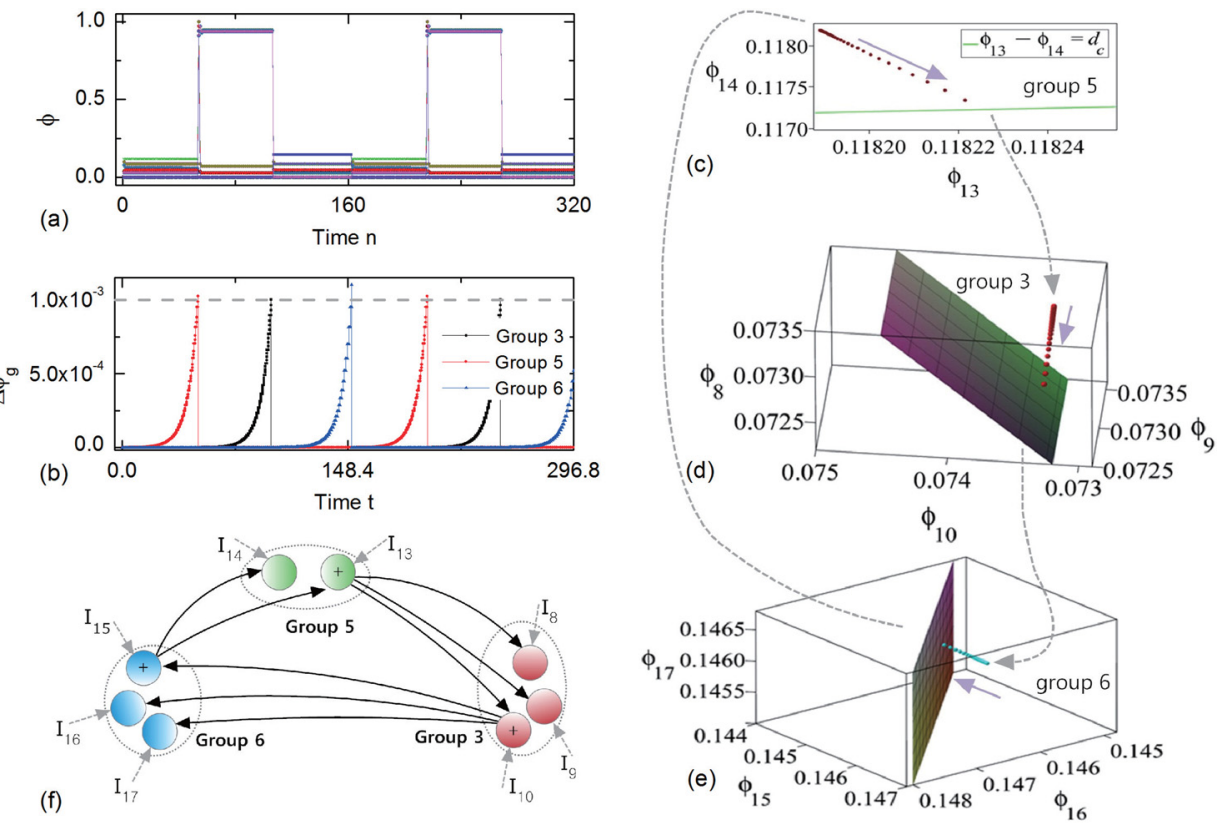

FIG. 3. Controlled generation of switching dynamics. The simulated pulse-coupled network has $N=30$ oscillators and 11 groups (parameters: $D=10^{-7}, d_{c}$ $=0.001, b=1.5, \varepsilon=0.1$, and $\tau=0.03$ ). (a) A switching pattern among three metastable states. The phases of all oscillators are plotted with respect to the $n$th resetting of oscillator 1. (b) Time evolution of the maximum phase difference $\Delta \phi_{g}$ for the three groups $(3,5$, and 6$)$ where they play the role of active firing groups for the three metastable states, respectively. (c)-(e) Demonstration of switching dynamics in low-dimensional subspaces where the dynamics is such that the phases of the active firing oscillators change fast, while those of the passive firing oscillators change slowly near the metastable states. Panel (c) shows some trajectory points $\left(\phi_{13}, \phi_{14}\right)$, with the green line indicating the critical condition (12) determined by $\phi_{13}-\phi_{14}=d_{c}$. Panels (d) and (e) show the slow subspace dynamics for the second and the third metastable states, respectively, and the two planes indicate the critical condition (12). The solid arrows indicate the evolution direction of the system, and the dashed arrows denote the jumps between different subspaces under localization control. In panel (f), only the connections among the three groups 3,5, and 6 are shown. The plus sign denotes the oscillator with the largest phase velocity in the same group.

symmetry-breaking parameters. The former can make the phase difference grow as $\exp (\varepsilon b)$. We consider the contribution from the symmetry-breaking parameter $\delta_{i}$. The change in the phase difference due to symmetry breaking is $\left|\delta_{i}-\delta_{j}\right| \Delta t$, where $\Delta t<1$ is the time duration for one resetting of the reference oscillator. The maximum change in the phase difference is $s_{c}=\max _{i} \delta_{i}-\min _{i} \delta_{i}$. We then have $\Delta \phi_{g}^{n}=\Delta \phi_{g}^{n-1} \exp (\varepsilon b)+s_{c}$, where $\Delta \phi_{g}^{n}$ denotes the maximum phase difference for the active firing group right after the $n$th resetting of the reference oscillator, and $\Delta \phi_{g}^{0}=0$ corresponds to the initiation of the localization control (identical phase for each oscillator in the same group). We have

$$
\begin{aligned}
\Delta \phi_{g}^{n} & =\sum_{i=1}^{n-1} \exp (i \times \varepsilon b) s_{c}+s_{c} \\
& =s_{c} \frac{1+[\exp (n \varepsilon b)-\exp (\varepsilon b)]}{\exp (\varepsilon b)-1} .
\end{aligned}
$$

For $\Delta \phi_{g}^{n}=d_{c}$, the corresponding value of $n$ is the residence time $T_{s}$ of the metastable state

$$
T_{s}=\frac{1}{\varepsilon b} \ln \left(\frac{d_{c}}{s_{c}}[\exp (\varepsilon b)-1]+1\right)
$$

TABLE II. Detailed process of localization control for a switching between two metastable states with group 5 and group 3 as the active firing groups, respectively. Here, group 5 contains two oscillators: 13 and 14; Group 3 contains three oscillators: 8, 9, and 10. Only the phases of these oscillators are shown, as other oscillators fire passively and are not relevant to the switching. The phases and time $t$ are shown just after the last event in the $k$ th sequence of events, and up to ten decimal digits are used to identify the difference of phases in the same group. Only the passive firings near the active firings are shown, while other passive firings are neglected as "..." The localization control is triggered just after the 3rd sequence of events, where the maximum phase difference of the

\begin{tabular}{|c|c|c|c|c|c|c|c|}
\hline \multirow[b]{2}{*}{$\mathrm{k}$} & \multirow[b]{2}{*}{ Sequence of events } & \multirow[b]{2}{*}{ Time $\mathrm{t}$} & \multicolumn{5}{|c|}{ Phases of oscillators from group 5 and group 3} \\
\hline & & & 8 & 9 & 10 & 13 & 14 \\
\hline 1 & $\cdots R(1,2,3,4,5,6,7,28,29,30)-S(13)-S(14)-R(13) S(8,9,10)$ & 48.80055 & 0 & 0 & 0 & 0.030000002 & 0.029147909 \\
\hline 2 & $\cdots R(1,2,3,4,5,6,7,28,29,30)-S(13)-S(14)-R(13) S(8,9,10)$ & 49.73842 & 0 & 0 & 0 & 0.030000002 & 0.029009955 \\
\hline 3 & $\cdots R(20,21,22,23) S(18,19,26,27)$ & 49.76941 & 0.030990045 & 0.030990047 & 0.030990047 & 0.074295733 & 0.073267853 \\
\hline 4 & $-S(8,9,10)$ & 49.76941 & 0 & 0 & 0 & 0.939939997 & 0.939939997 \\
\hline 5 & $R(8,9,10) S(11,12,15,16,17,24,25)$ & 49.79941 & 0.0300000003 & 0.0300000024 & 0.0300000025 & 0.969939999 & 0.969939997 \\
\hline 6 & $\cdots S(10)-S(9)-S(8)-R(10) S(15,16,17)$ & 50.73973 & 0.029999930 & 0.029999997 & 0.030000002 & 0.949243612 & 0.949243558 \\
\hline 7 & $\cdots S(10)-S(9)-S(8)-R(10) S(15,16,17)$ & 51.68006 & 0.029999849 & 0.029999991 & 0.030000002 & 0.949243609 & 0.949243555 \\
\hline
\end{tabular}
group 5 with oscillators 13 and 14 is about 0.00102 which is larger than $d_{c}=0.001$. Group 3 with oscillators 8,9 , and 10 is selected as the first level group during the localization control and becomes immediately firing, i.e., the 4 th event. 
We see that $T_{s}$ can be adjusted by choosing appropriate parameters such as $\varepsilon$ and $b$ for fixed value of $d_{c}$ (e.g., $d_{c}$ $=0.001)$. We have $s_{c}=D\left(\max A_{i}-\min A_{i}\right)$ from Eq. (9). As $A_{i}$ is randomly chosen from the interval $[-0.5,0.5]$, we choose $s_{c}=D$. We can then adjust the parameters to realize the desired residence time, for example, $T_{s} \approx 49.3$ for $b=$ $1.5, \varepsilon=0.1, d_{c}=0.001$, and $D=10^{-7}$.

The saddle residence time $T_{s}$ can be used to computationally detect the metastable states. We first locate a trajectory of length $L$, where the $m$ th trajectory point is the state of the system immediately after the $m$ th resetting of the reference oscillator 1 at time $t_{m}$. For such a state, the phase of oscillator $i$ is $\phi_{i}\left(t_{m}\right)$. The distance $E$ between two trajectory points, e.g., the $m$ th and $n$th points with time $t_{m}$ and $t_{n}$, respectively, is defined as

$$
E=\sum_{i}\left|\phi_{i}\left(t_{m}\right)-\phi_{i}\left(t_{n}\right)\right| .
$$

This quantity can be used to locate the metastable states. In particular, according to the property of metastable states, if the system can stay near a tested state for at least $T_{m}$ number of resettings of the reference oscillator, i.e., with corresponding distance $E<E_{m}$, the tested state is regarded as a metastable state. In practice, some successive points may satisfy the above condition. In such a case, we choose the middle point as the metastable state.

We address how to choose $T_{m}$ and $E_{m}$ using the residence time $T_{s}$ and the departure process of the system from a metastable state. Because of the transient nature of the system dynamics near a metastable state after application of localization control, we choose

$$
T_{m}=0.5 \times T_{s},
$$

to make the algorithm robust.

The value of $E_{m}$ can be chosen as the critical distance between the metastable state and the state immediately before the maximum phase difference for a group exceeds $d_{c}$. Both active and passive firing oscillators contribute to $E_{m}$. We first consider the active firing oscillators. As the maximum phase difference of this firing group grows exponentially, the phase of the oscillator with the largest phase velocity (leading oscillator) typically becomes much larger than those of the other oscillators. The contribution to the value of $E_{m}$ due to the leading oscillator is then about $d_{c}$. Suppose that there are $N_{a}$ active firing oscillators. Their contribution is less than $N_{a} d_{c}$. We next analyze the contribution from the passive firing oscillators. For them, only the phases of the oscillators receiving pulses from the active firing oscillators are affected, and these pulses will arrive within $d_{c}$ time, because all oscillators in the active firing group will be reset within this time window. The number of such affected oscillators is smaller than $N-N_{a}$, because not all oscillators are connected to the active firing group. For each affected passive firing oscillator, we have $U^{-1}\left[U\left(\phi+d_{c}\right)+\varepsilon^{\prime}\right]$ $-U^{-1}\left[U(\phi)+\varepsilon^{\prime}\right] \approx d_{c}$ for small values of $\varepsilon^{\prime}$. We can then approximate the threshold distance as

$$
E_{m}=N_{a} d_{c}+\left(N-N_{a}\right) d_{c}=N d_{c} .
$$

Numerically we find that $T_{m}$ and $E_{m}$ so chosen can result in reliable identification of the metastable states.

\section{Controlled generation of switching dynamics in networks of varying structures}

We are interested in the performance of the localization control method in various networks with groups. We thus introduce a measure, $f_{\mathrm{LC}}$, to quantify how well the switching between two successive metastable states is determined by localization control, which is averaged over different network realizations. Specifically,

$$
f_{\mathrm{LC}}=\frac{1}{N_{1} \times N_{2}} \sum_{i}^{N_{1}} \sum_{j}^{N_{2}} f_{i j},
$$

where $N_{1}$ is the number of network realizations and for each we test $N_{2}$ number of trajectories with length of 1000 resettings of the reference oscillator 1 . Then, for the $j$ th trajectory of the network $i$, we calculate the fraction $f_{i j}$ of switchings between any two successive metastable states that are successfully determined by the localization control method (The detailed calculation is described later in this section.). For each network, we use different phase velocity distributions. For each trajectory, a randomly chosen group is used as the first level group to initiate localization control. In this way, many different switching patterns may be generated. We then construct $N_{1}$ different networks with given topological parameters such as number of oscillators for one group or the in-degree. The detailed construction can be found in the Appendix. The case of $f_{\mathrm{LC}}=1$ indicates that the switching dynamics is fully determined by the network structures when localization control is applied.

We describe the detailed process to obtain $f_{i j}$ for $j$ th trajectory in $i$ th network, with length of 1000 resettings of the reference oscillator 1 . We first locate all metastable states. We then obtain the event property of each group during one resetting of the reference oscillator at a metastable state to determine whether it has the event structure of one active firing group and other groups with passive firing. As the system may switch on its own from one metastable to another state, we determine whether the switching between two successive metastable states is realized through localization control using the winner-based rule. For a trajectory exhibiting $m$ metastable states, we test all $(m-1)$ switchings between two successive metastable states. The fraction of switchings determined by the localization method is thus $f_{i j}=n /(m-1)$, where $n$ denotes the number of switchings correctly realized by the winner-based rule. In the cases of $m$ $=0$ or 1 , we let $f_{i j}=0$ because no switching occurs.

We calculate the dependence of $f_{\mathrm{LC}}$ on the parameters $(\tau, \varepsilon)$ for networks of $N=30$ oscillators, where each oscillator within a group points to only one group, and the number of oscillators for each group is chosen from 2 to 4 . The other parameters are $b=1.5$ and $d_{c}=0.001$, and $\delta_{i}$ is randomly and uniformly chosen from $D[-0.5,0.5]$ with $D=10^{-7}$. The residence time is calculated according to Eq. (14) with $s_{C}$ $=D$. Figure 4 (a) shows the result $f_{\mathrm{LC}}$. We see a region in the lower left corner of the $(\tau, \varepsilon)$ plane with $f_{\mathrm{LC}}=1$, indicating a 
correspondence between network structures and switching dynamics under the localization control. In addition, the success of localization control mainly depends on the parameter $\tau$, whose effect is analyzed later in this section.

We also study the case where each oscillator can point to more than one group. We use the in-degree $k_{i}$, i.e., the number of incoming links of an oscillator $i$, to quantify the network topology. We consider the networks in which all oscillators have the same in-degree. We calculate the dependence of $f_{\mathrm{LC}}$ on the parameters $\tau$ and in-degree $k_{i}$ for relatively large
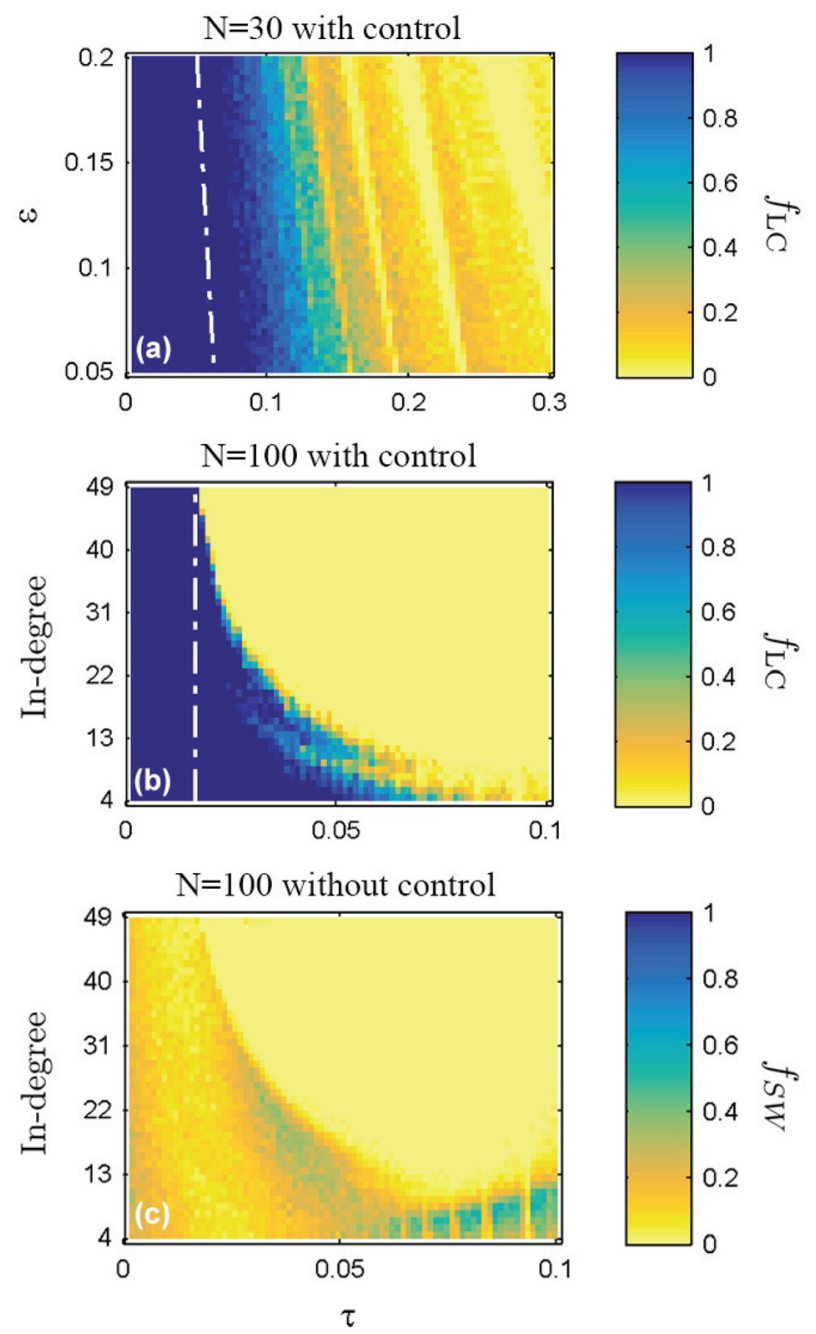

FIG. 4. Interplay between switching dynamics and network structures. (a) The contour plot of the fraction $f_{L C}$ of switchings determined by the localization control in the parameter plane $(\tau, \varepsilon)$, where networks are of size $N=30$ and each oscillator points to only one group of oscillators. The number of oscillators in a group is from 2 to 4 . (b) and (c) The switching dynamics for networks of size $N=100$ and each group with two oscillators, where each oscillator $i$ has the same in-degree $k_{i}$. (b) Contour plot of $f_{L C}$ in the parameter plane $(\tau$, In - degree) for $\varepsilon=0.15$. The dashed-dotted line indicates the estimated critical line for the correspondence between structures and switchings. (c) Switching dynamics for networks of 100 oscillators with the same setup as in (b), but without control. Contour plot of $f_{S W}$, the fraction of trajectories with switching dynamics, shows that the switching behavior is quite rare without control. The corresponding value for each parameter point is averaged over 30 independent network realizations, each with 100 trajectories containing 1000 resettings of oscillator 1. For each network, the symmetry-breaking parameters are randomly chosen to realize various distinct switching patterns. Other parameters are $b=1.5, d_{c}=0.001$, and $D=10^{-7}$. networks by fixing $\varepsilon=0.15$. For example, we consider networks of $N=100$ oscillators divided into 50 groups, where the in-degree $k_{i}$ varies from 4 to 49 , corresponding to change in the network structure from sparse to globally connected in terms of groups. We find that our control method can sustain the structure-dynamics correspondence for a variety of network structures, as exemplified in Fig. 4(b).

To compare the results with control, we study switching dynamics in networks of size 100 under the same parameter setting as in Fig. 4(b) but without control. For a trajectory starting from a random initial condition, we determine whether the switching phenomena persist, i.e., there are at least two metastable states. As no control is present, we use a new measurement, the fraction of trajectories with switching phenomena, $f_{\mathrm{sw}}$, which is obtained by averaging over 30 network realizations and 100 trajectories. As shown in Fig. 4(c), the fraction $f_{\mathrm{sw}}$ is small, indicating that switching becomes rare in absence of control. This is because the phase space is dominated by the basins of the stable attractors when links are removed from the configuration of globally connected network. Without control, the system quickly approaches one of the stable attractors, terminating the switching dynamics. We also find that, for sparse networks, sustained switching behavior is uncommon. In these cases, control is absolutely necessary to generate any desired switching dynamics.

\section{Correspondence between network structure and switching dynamics}

The results in Fig. 4 suggest that there exists a parameter region in which a correspondence between the network structure and switching dynamics exists, especially for relatively small values of $\tau$ and $\varepsilon$. For the localization control, groups are organized into different levels, which determine the initial condition according to Eq. (10). Success of localization control requires that the chosen initial condition can lead to a metastable state, and the metastable state can be sustained under the given parameters.

According to Fig. 4(b), the critical value of $\tau$ for successful localization control is smaller for networks with larger indegrees. Thus, we first study globally connected networks in terms of groups. When applying the localization control, the groups are organized into two levels with one group (denoted by $g_{a}$ ) in the first level and all other groups (denoted by $g_{o}$ ) in the second level. For a metastable state, the first level group fires actively, and the groups in the second level fire passively. Figure 5 shows typical events associated with the evolution of the system after applying control at time 0 . We first consider the active firing group $g_{a}$ and calculate the phase $\phi_{a f}^{3}$ of an active firing oscillator in group $g_{a}$ just after the 3rd event $R\left(g_{o}\right)$. Immediately preceding this event, the phase of the chosen active firing oscillator is $2 \tau$, and all the pulses from its neighboring oscillators are received. The phase $\phi_{\text {af }}^{3}$ just after the 3rd event then becomes

$$
\phi_{\text {af }}^{3}=H(2 \tau, \varepsilon),
$$

where 


$$
H\left(\phi, \varepsilon^{\prime}\right)=U^{-1}\left[U(\phi)+\varepsilon^{\prime}\right]
$$

represents the response of an oscillator of phase $\phi$ to a subthreshold pulse of strength $\varepsilon^{\prime}$, i.e., $U(\phi)+\varepsilon^{\prime}<1$. Just after the 3rd event, an active firing oscillator needs a time duration $\Delta w$ to reach the threshold

$$
\Delta w=1-H(2 \tau, \varepsilon) .
$$

We then consider the passive firing groups denoted by $g_{o}$. Each group has directed links from one oscillator in each other group. The strength of each incoming link is $\varepsilon /\left(N_{g}-1\right)$, where $N_{g}$ denotes the number of groups. The phase of a passive firing oscillator is reset at the 2nd event. At the 3rd event, each passive firing oscillator receives $\left(N_{g}-2\right)$ pulses with the strength

$$
\varepsilon_{3}=\varepsilon \frac{N_{g}-2}{N_{g}-1}=\varepsilon\left[1-\frac{1}{N_{g}-1}\right] .
$$

The phase $\phi_{p f}^{3}$ just after 3rd event is

$$
\phi_{p f}^{3}=H\left(\tau, \varepsilon_{3}\right) .
$$

After that, the passive firing oscillator undergoes free evolution of duration $\Delta w+\tau$, and receives one pulse from $g_{a}$, i.e., the 5 th event. The strength of the pulse is

$$
\varepsilon_{5}=\varepsilon /\left(N_{g}-1\right) .
$$

The phase of the chosen passive firing oscillator just before the 5th event is $H\left(\tau, \varepsilon_{3}\right)+\Delta w+\tau$. Then, the occurrence of passive firing requires

$$
U\left(H\left(\tau, \varepsilon_{3}\right)+\Delta w+\tau\right)+\varepsilon_{5}>1 .
$$

Equation (25) can be used to estimate the parameter region in which there is a direct correspondence between the network structure and the switching dynamics. As shown in Fig. 4(a), the dashed-dotted line indicates the critical line in the $(\tau, \varepsilon)$ plane for $N_{g}=15$ and $N=30$. The dynamical behaviors associated with the parameter plane $(\tau$, In-degree) are shown in Fig. 4(b), where the dashed-dotted curve is for $N_{g}$ $=50$ and $\varepsilon=0.15$.

Temporal distribution of the pulses for an oscillator can affect its phase. If more pulses are received during the time

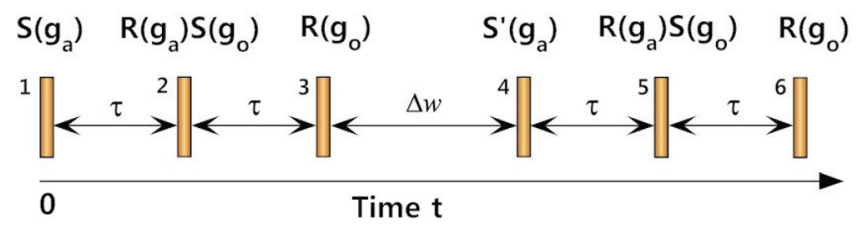

FIG. 5. Typical events associated with system evolution after applying the localization control at time 0 for globally connected networks in terms of groups. The first level group is $g_{a}$, which becomes immediately firing denoted by $S\left(g_{a}\right)$. After a free evolution of time duration $\tau$, the pulses from group $g_{a}$ are received, immediately making other groups (denoted by $g_{o}$ ) fire passively with the event $R\left(g_{a}\right) S\left(g_{o}\right)$. After that, a time duration of $\Delta w$ is needed for the firing of group $g_{a}$. Due to symmetry breaking, the oscillators in group $g_{a}$ fire at slightly different times. For simplicity, we denote these firings as $S^{\prime}\left(g_{a}\right)$. when the phase of the oscillator is small, the contribution of these pulses to the phase will be smaller, because $H\left(\phi, \varepsilon^{\prime}\right)$ is a monotonous function of $\phi$ with a positive slope. As an example, we consider networks of even size $N$ which are strongly connected in terms of groups. Each group has two oscillators, so the number of groups is $N_{g}=N / 2$. A passive firing oscillator receives pulses at the $3 \mathrm{rd}$ and the 5 th events, as shown in Fig. 5, whose strengths are $\varepsilon_{3}$ and $\varepsilon_{5}$, respectively. As the number of groups is increased, $\varepsilon_{3}$ and $\varepsilon_{5}$ become larger and smaller, respectively, so the phase immediately preceding the 5 th event is smaller, leading to a smaller critical $\tau$ for localization control. This is because that the right term of Eq. (25) is decreasing with respective to $\tau$. The result is shown in Fig. 6, where the dashed-dotted line indicates the critical line determined from Eq. (25).

For a sparse network, the groups can be organized with the number of levels much larger than two. In such a case, a passive firing oscillator can receive pulses from the groups with the level number higher than 2, where the corresponding phase is much larger than that in a globally coupled network. As a result, the critical value of $\tau$ for control is also larger.

In the above analysis, we locate the parameter region in which a correspondence between switching dynamics and network structure exists. In such a case, a switching between two successive metastable states is due to the directed links between two specific groups, i.e., two active firing groups, which are associated with the two metastable states, respectively. When more groups are taken into account, a loop structure can emerge, which can in turn induce a stationary switching pattern, as shown in Fig. 3. Generally, the loops at the group level cause stable switching patterns. The number of different stable switching patterns determines the computational capacity of the system, which is then determined by the number of loops. The number of loops tends to grow quickly with the link density. The loop structures can then be exploited to realize desired switching patterns.

As an illustrative example, we study a network of $N=9$ oscillators, organized into 4 groups as shown in Fig. 7(a).

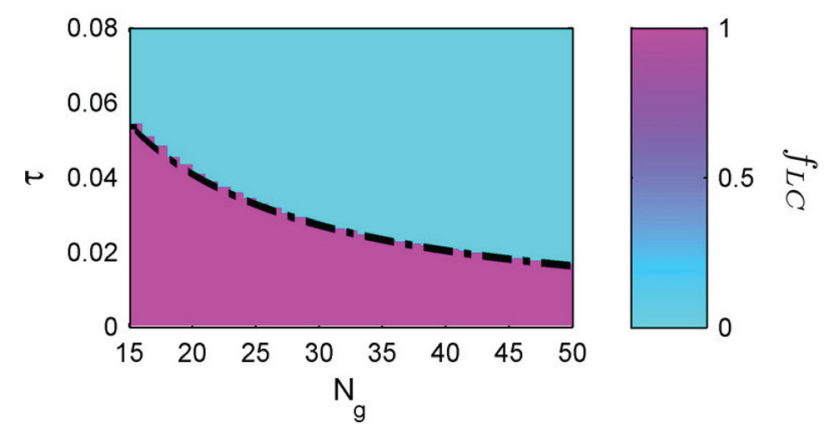

FIG. 6. Estimated parameter region where switching is successfully controlled by the localization method. Dependence of $f_{L C}$ on parameter $\tau$ and the number of groups $N_{g}$. The networks are globally connected in terms of groups where each group receives links from all other groups, and each group has two oscillators. Thus, the network size is $N=2 N_{g}$. The dasheddotted line indicates the critical line for the correspondence between structure and switching dynamics. The value of $f_{L C}$ of each parameter point is obtained through averaging over the results of 100 trajectories. 
There are various loops of length 2,3, and 4 which is defined as the number of involved groups. For all group pairs, there are three loops, denoted as $L_{2 a}, L_{2 b}$, and $L_{2 c}$, respectively. For loops of length 3, there are four loops: $L_{3 a}, L_{3 b}, L_{3 c}$, and $L_{3 d}$. For the loops of length 4 , for clarity only one loop $L_{4 a}$ (the one formed by the green links) is shown in Fig. 7(a). We set the parameters to be $D=10^{-7}, \tau=0.05, \varepsilon=0.1$, and $b$ $=1.5$, under which there exists one-to-one correspondence between the switching dynamics and the network structure, as Eq. (25) predicts. The correspondence between switching patterns and the loop structures is shown in Figs. 7(b)-7(d), where appropriately leading oscillators of the groups are

(a)
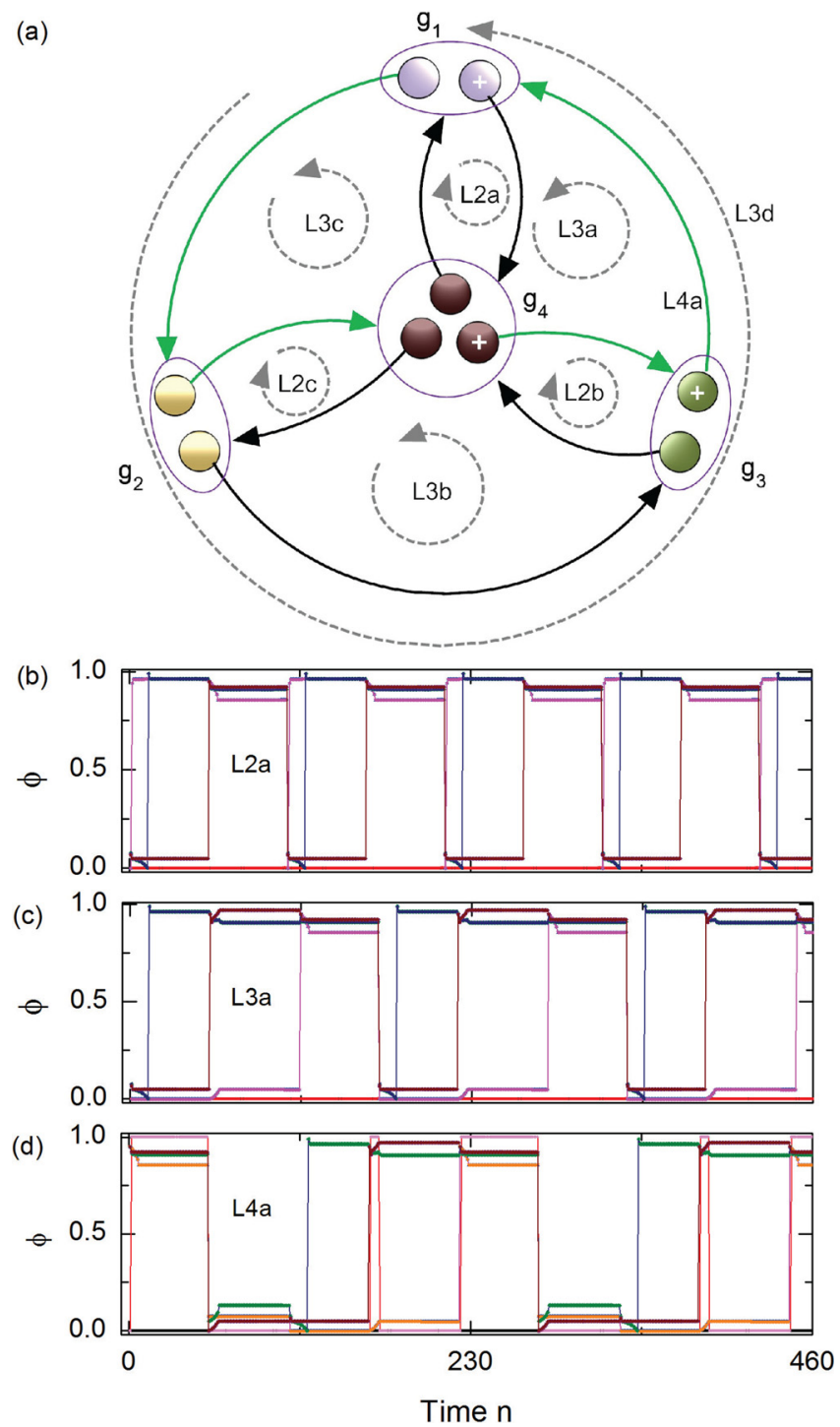

FIG. 7. Switching patterns and the loops of groups. For $\tau=0.05, \varepsilon=0.1$, and $b=1.5$, (a) a network with four groups $\left(g_{1}, g_{2}, g_{3}\right.$, and $\left.g_{4}\right)$, where each directed link represents excitatory coupling from the starting oscillator to a group of oscillators. The groups can form loops denoted by the dashed curved arrows. The notion $L 2_{a}$, for example, represents a loop of length 2 (two groups). (b)-(d) The corresponding stable switching patterns with loops $L_{2 a}, L_{3 a}$, and $L_{4 a}$, where the length is from 2 to 4 at the group level, respectively. The proper choice of the leading oscillators (denoted by symbol "+") in the three groups leads to the loop $L_{3 a}$ as shown in (a), which in turn is responsible for the switching pattern occurring among the three metastable states shown in (c). used to form loops. This can be realized by selecting the required leading oscillator for a group $g$. Specifically, we can assign maximum phase velocity among some randomly generated phase velocities to an oscillator to make it a leading oscillator for a group. The loop $L_{3 a}$ is formed if we choose three leading oscillators denoted by the plus sign "+" for corresponding three groups, respectively, as shown in Fig. 7(a). Accordingly, a switching pattern among the three metastable states can be realized, as shown in Fig. 7(c), where any of the involved three groups can be chosen as the starting group to initiate localization control. In the similarly way, we can realize the other three $L_{3}$ types of switching behaviors, each of which is the result of a loop induced by different leading oscillators.

\section{CONCLUSIONS}

Unstable saddles represent a fundamental class of invariant sets in nonlinear dynamical systems. For a highdimensional network system, saddles can appear in large numbers. It is appealing, from the perspective of information science and technology, to generate trajectories with multiple metastable states and switchings among them. Without control, a nonlinear system is typically not able to generate such trajectories, due to the existence of stable attractors. Controlled generation of switching dynamics among saddles is thus of interest.

In this paper, utilizing networks of pulse-coupled oscillators as a paradigm, we present an intuitive but feasible approach to realizing switching dynamics for a wide variety of network structures. A key is the existence of a class of saddles with common event properties, enabling us to use localization control to direct the whole network to switch from one metastable state to another. As a result, establishing direct heteroclinic connections among saddles is not necessary, which is generally a difficult task for high dimensional dynamical systems. We uncover a correspondence between network structure and switching dynamics, which allows us to harness the switching dynamics in phase space through manipulating groups of oscillators. In particular, we can generate desired stationary switching dynamics through designing the loop structures at the level of groups.

We remark that, while the starting point of our work is the Mirollo-Strogatz model, our interest is in event-based dynamics where the resulting $U(x)$ function is a transformation from the original differential equation representation. In this sense, our setting goes beyond that in the original MirolloStrogatz model. We mention that certain one-dimensional models, such as the leaky integrate-and-fire model and the $\Theta$ neuron, can be transformed to the Millro-Strogatz model through specific nonlinear transformation of variables. Our strategy of controlled generation of desired switching dynamics can be straightforwardly applied to these models.

The inputs can be properly scaled to be small values and added to the parameters. Thus, our method can be used to process input information based on the input-driven switching dynamics. One advantage is that networks structures can be readily designed to yield desired switching dynamics. Besides, we can include more features into the method such 
as the hierarchical organization of networks, where a group can contain several sub-groups. How to make the generation of switching dynamics robust to noise is also a topic worth further investigating.

The switching dynamics is realized through the network structure and the control rule. Here, we consider one type of "winner" rules where the oscillator with the largest phase velocity is the most important. Some other rules are also possible, such as those depending on the specific values of the phase velocities of the oscillators, which can increase the computational capability to process inputs. Another open issue is to change the ways of localization when the number of saddles and accordingly the number of switching patterns become much larger.

\section{ACKNOWLEDGMENTS}

This research was supported by the Aihara Project, the FIRST program from JSPS, initiated by CSTP, and CREST, JST. Y.C.L. was supported by ARO under Grant No. W911NF-14-1-0504. Z.C.D. was supported by the National Natural Science Foundation of China (No. 11432010). H.L.Z. was supported by "The Fundamental Research Funds for the Central Universities" (No. 3102014JCQ01036), and by the National Natural Science Foundation of China (No. 11502200). We also thank anonymous reviewers for their insightful and useful comments.

\section{APPENDIX: CONSTRUCTION OF NETWORKS WITH GROUPS}

We first organize oscillators into groups with the requirement that each group has at least two oscillators. We then put the directed links among the oscillators with the requirement that oscillators within the same group receive the same set of incoming links. There are three additional requirements. The first is that each oscillator points to at least one group. In this case, we can always select a group during the localization control for any leading oscillator. The second is that we avoid the case that multiple oscillators in a group point to a same group, as the choice of different leading oscillators can lead to the same group in such a case. The third is that the networks are strongly connected in terms of groups; i.e., each group is reachable from every other group through the directed links. This requirement is imposed to ensure that any group can be used as the first level group.

Throughout this paper, we consider two types of networks. For the first type, each oscillator within a group points to only one group. We place directed links from oscillator $i$ to all the oscillators in a randomly selected group $g$, which does not contain oscillator $i$. For this type of networks, the allowed maximum number of oscillators within a group is $N_{g}-1$, where $N_{g}$ is the number of groups.

For the second type, each oscillator $i$ has the same indegree $k_{i}$ and is allowed to point to multiple groups. As multiple oscillators in a group are not allowed to point to a same group, the in-degree is $k_{i} \leq\left(N_{g}-1\right)$. For group $g$, we first randomly select $k_{i}$ groups from other groups. Then for each selected group, we randomly select an oscillator $i$ in that group and place directed links from $i$ to all oscillators in the group $g$. A particular example for this type of networks is globally connected networks in terms of groups, where each oscillator in a group receives links from one randomly chosen oscillator from each other group. In such a case, the indegree $k_{i}$ for each oscillator $i$ is $k_{i}=N_{g}-1$.

${ }^{1}$ J. J. Hopfield, Proc. Nat. Acad. Sci. U.S.A. 79, 2554 (1982).

${ }^{2}$ K. Aihara, T. Takabe, and M. Toyoda, Phys. Lett. A 144, 333 (1990).

${ }^{3}$ T. Nishikawa, Y.-C. Lai, and F. C. Hoppensteadt, Phys. Rev. Lett. 92, 108101 (2004).

${ }^{4}$ S. Sihna and W. L. Ditto, Phys. Rev. Lett. 81, 2156 (1998).

${ }^{5}$ S. Sinha, T. Munakata, and W. L. Ditto, Phys. Rev. E 65, 036214 (2002).

${ }^{6}$ M. Jahed-Motlagh, B. Kia, W. L. Ditto, and S. Sinha, Int. J. Bifurcation Chaos 17, 1955 (2007).

${ }^{7}$ W. L. Ditto, K. Murali, and S. Sinha, Philos. Trans. R. Soc. London, Ser. A 366, 653 (2008).

${ }^{8}$ G. Laurent, M. Stopfer, R. W. Friedrich, M. I. Rabinovich, and H. D. I. Abarbanel, Ann. Rev. Neurosci. 24, 263 (2001).

${ }^{9}$ M. Rabinovich, R. Huerta, and G. Laurent, Science 321, 48 (2008).

${ }^{10}$ W. Maass, T. Natschläger, and H. Markram, Neural Comput. 14, 2531 (2002).

${ }^{11}$ H. Jaeger and H. Haas, Science 304, 78 (2004).

${ }^{12}$ D. V. Buonomano and W. Maass, Nat. Rev. Neurosci. 10, 113 (2009).

${ }^{13} \mathrm{U}$. Alon, An Introduction to Systems Biology (Chapman and Hall/CRC, London, 2007).

${ }^{14}$ E. Ott, Chaos in Dynamical Systems, 2nd ed. (Cambridge University Press, Cambridge, UK, 2002).

${ }^{15}$ Y.-C. Lai and T. Tél, Transient Chaos: Complex Dynamics on Finite Time Scales (Springer, New York, 2011).

${ }^{16}$ K. Keplinger and R. Wackerbauer, Chaos 24, 013126 (2014).

${ }^{17}$ E. Ott, C. Grebogi, and J. A. Yorke, Phys. Rev. Lett. 64, 1196 (1990).

${ }^{18}$ S. Boccaletti, C. Grebogi, Y.-C. Lai, H. Mancini, and D. Maza, Phys. Rep. 329, 103 (2000).

${ }^{19} \mathrm{G}$. Chen, Controlling Chaos and Bifurcations in Engineering Systems, 1st ed. (CRC Press, Boca Raton, Florida, 1999).

${ }^{20}$ M. Rabinovich, A. Volkovskii, P. Lecanda, R. Huerta, H. D. I. Abarbanel, and G. Laurent, Phys. Rev. Lett. 87, 068102 (2001).

${ }^{21}$ P. Ashwin, G. Orosz, and S. Townley, SIAM J. Appl. Dyn. Syst. 6, 728 (2007).

${ }^{22}$ F. Schittler Neves and M. Timme, J. Phys. A: Math. Theor. 42, 345103 (2009).

${ }^{23}$ M. K. Muezzinoglu, I. Tristan, R. Huerta, V. S. Afraimovich, and M. I. Rabinovich, Int. J. Bifurcation Chaos 20, 1653 (2010).

${ }^{24}$ F. Schittler Neves and M. Timme, Phys. Rev. Lett. 109, 018701 (2012).

${ }^{25}$ P. Ashwin and C. Postlethwaite, Physica D 265, 26 (2013).

${ }^{26}$ M. A. Komarov, G. V. Osipov, and C. S. Zhou, Phys. Rev. E 87, 022909 (2013).

${ }^{27}$ M. I. Rabinovich, P. Varona, I. Tristan, and V. S. Afraimovich, Front. Comput. Neurosci. 8, 22 (2014).

${ }^{28}$ O. V. Maslennikov and V. I. Nekorkin, Commun. Nonlinear Sci. Numer. Simul. 23, 10 (2015).

${ }^{29}$ V. S. Afraimovich, V. P. Zhigulin, and M. I. Rabinovich, Chaos 14, 1123 (2004).

${ }^{30} \mathrm{P}$. Ashwin, O. Karabacak, and T. Nowotny, J. Math. Neurosci. 1, 13 (2011).

${ }^{31}$ M. Krupa, J. Nonlinear Sci. 7, 129 (1997).

${ }^{32}$ M. Timme, F. Wolf, and T. Geisel, Phys. Rev. Lett. 89, 154105 (2002).

${ }^{33}$ M. Timme, F. Wolf, and T. Geisel, Chaos 13, 377 (2003).

${ }^{34}$ P. Ashwin and M. Timme, Nonlinearity 18, 2035 (2005).

${ }^{35}$ P. Varona, M. I. Rabinovich, A. I. Selverston, and Y. I. Arshavsky, Chaos 12, 672 (2002).

${ }^{36}$ A. T. Winfree, The Geometry of Biological Time, 2nd ed. (Springer, New York, 2000).

${ }^{37}$ R. E. Mirollo and S. H. Strogatz, SIAM J. Appl. Math. 50, 1645 (1990).

${ }^{38}$ M. Tsodyks, I. Mitkov, and H. Sompolinsky, Phys. Rev. Lett. 71, 1280 (1993).

${ }^{39}$ C. V. Vreeswijk, Phys. Rev. E 54, 5522 (1996).

${ }^{40}$ W. Gerstner, Phys. Rev. Lett. 76, 1755 (1996).

${ }^{41}$ D. Z. Jin, Phys. Rev. Lett. 89, 208102 (2002).

${ }^{42}$ A. Zumdieck, M. Timme, T. Geisel, and F. Wolf, Phys. Rev. Lett. 93, 244103 (2004). 
${ }^{43}$ P. Gong and C. van Leeuwen, Phys. Rev. Lett. 98, 048104 (2007).

${ }^{44}$ S. Jahnke, R.-M. Memmesheimer, and M. Timme, Phys. Rev. Lett. 100, $048102(2008)$.

${ }^{45}$ R. Zillmer, N. Brunel, and D. Hansel, Phys. Rev. E 79, 031909 (2009).

${ }^{46}$ H. Zou, X. Gong, and C.-H. Lai, Phys. Rev. E 82, 046209 (2010).

${ }^{47}$ C. Kirst and M. Timme, Phys. Rev. E 78, 065201 (2008).
${ }^{48}$ C. Kirst, T. Geisel, and M. Timme, Phys. Rev. Lett. 102, 068101 (2009).

${ }^{49}$ J. Milnor, Commun. Math. Phys. 102, 517 (1985).

${ }^{50}$ H.-L. Zou, M. Li, C.-H. Lai, and Y.-C. Lai, Phys. Rev. E 86, 066214 (2012).

${ }^{51}$ H. Broer, K. Efstathiou, and E. Subramanian, Nonlinearity 21, 1385 (2008). 